\title{
Association Between Ethnicity and Severe COVID-19 Disease: a Systematic Review and Meta-analysis
}

\author{
Antony Raharja ${ }^{1} \cdot$ Alice Tamara $^{2} \cdot$ Li Teng Kok $^{1}$ \\ Received: 29 August 2020 / Revised: 29 August 2020 / Accepted: 1 November 2020 / Published online: 12 November 2020 \\ (C) W. Montague Cobb-NMA Health Institute 2020
}

\begin{abstract}
Objectives This article evaluates if ethnicity is an independent poor prognostic factor in COVID-19 disease.

Methods MEDLINE, EMBASE, Cochrane, WHO COVID-19 databases from inception to 15/06/2020 and medRxiv. No language restriction. Newcastle-Ottawa Scale (NOS) and GRADE framework were utilised to assess the risk of bias and certainty of evidence. PROSPERO CRD42020188421.

Results Seventy-two articles (59 cohort studies with 17,950,989 participants, 13 ecological studies; 54 US-based, 15 UK-based; 41 peer-reviewed) were included for systematic review and 45 for meta-analyses. Risk of bias was low: median NOS 7 of 9 (interquartile range 6-8). Compared to White ethnicity, unadjusted all-cause mortality was similar in Black (RR: 0.96 [95\% CI: 0.83-1.08]) and Asian (RR: 0.99 [0.85-1.16]) but reduced in Hispanic ethnicity (RR: 0.69 [0.57-0.84]). Age- and sex-adjusted risks were significantly elevated for Black (HR: 1.38 [1.09-1.75]) and Asian (HR: 1.42 [1.15-1.75]), but not for Hispanic (RR: 1.14 [0.93-1.40]). Further adjusting for comorbidities attenuated these associations to non-significance: Black (HR: 0.95 [0.721.25]); Asian (HR: 1.17 [0.84-1.63]); Hispanic (HR: 0.94 [0.63-1.44]). Subgroup analyses showed a trend towards greater disparity in outcomes for UK ethnic minorities, especially hospitalisation risk.

Conclusions This review could not confirm a certain ethnicity as an independent poor prognostic factor for COVID-19. Racial disparities in COVID-19 outcomes may be partially attributed to higher comorbidity rates in certain ethnicity.
\end{abstract}

Keywords Ethnicity $\cdot$ COVID-19 $\cdot$ Mortality $\cdot$ Hospitalisation $\cdot$ Acute kidney injury $\cdot$ Intubation

\section{Introduction}

Up to $85 \%$ of individuals affected by SARS-CoV-2 are asymptomatic or have mild illness, $15 \%$ require oxygen and $5 \%$ critically ill requiring intensive care unit (ICU) admission [1]. Several risk factors such as age, male sex and comorbidities have been shown to correlate with more severe disease

Antony Raharja, Alice Tamara and Li Teng Kok are equally contributing co-first authors.

\section{Highlights}

- Ethnicity is not an independent poor prognostic factor in COVID-19 disease.

- Racial disparities in COVID-19 outcomes can be attributed to higher rates of medical comorbidities in ethnic minority groups.

- Disproportionate impact of COVID-19 on ethnic minorities is possibly largely driven by increased infection rates amongst ethnic minorities.

\section{Antony Raharja}

antony.raharja@nhs.net

1 Guy's and St Thomas' NHS Foundation Trust, London, UK

2 Faculty of Medicine, Universitas Indonesia, Jakarta, Indonesia
[2]. Understanding the demographic risk factors for severe disease is of paramount importance not only to inform clinical practice but also to inform risk stratification at workplace and focus public health efforts at protecting vulnerable groups and those most affected by COVID-19 [3].

Multiple studies reported overrepresentation of ethnic minority groups in COVID-19 deaths and ICU admissions [4, 5]. A previous systematic review by Pan et al. highlighted the lack of ethnicity reporting in the literature. There was a consistent evidence of greater infection rates amongst ethnic minorities, but it noted a conflicting evidence on the relationship between ethnicity and mortality [6]. The association between ethnicity and COVID-19 outcomes may be riddled with confounding factors such as age, sex, comorbidities and socioeconomic factors [7]. As such, considerations of covariates are necessary to determine if ethnicity is truly an independent risk factor for poor outcomes in COVID-19.

This systematic review and meta-analysis evaluates the association between ethnicity and poor outcomes (mortality, hospitalisation, ICU admission, advanced respiratory support and kidney failure) in patients with laboratory-confirmed SARS-CoV-2. 


\section{Method}

This study was conducted according to the Preferred Reporting Items for Systematic Review and Meta-Analysis (PRISMA) Statement [8]. The protocol of this review was registered in PROSPERO database, CRD42020188421.

\section{Data Sources and Searches}

A literature search of databases was conducted from database inception to 31 May 2020, and later updated on 15 June 2020. Databases used were Ovid MEDLINE, Ovid EMBASE, Cochrane COVID-19 Study Register and World Health Organization (WHO) COVID-19 Global Research Database. Medical Subject Headings (MeSH) terms were 'SARS-CoV-2 OR Covid-19 OR novel coronavirus' and 'ethnic OR race OR minority group OR demography'. The Lancet, BMJ and JAMA were reviewed for any relevant articles. Preprint servers (MedRxiv and BioRxiv) were searched for non-peer-reviewed preprint articles. References of included studies and a related systematic review were screened for relevant articles as part of literature snowballing [6]. Citation tracking was carried out using Google Scholar on 24 June 2020 to identify relevant articles which cited any of the included studies. No language restrictions were applied. All steps were carried out by pairs of independent reviewers (AT, LK, AR), and a third reviewer arbitrated for cases without consensus. Full details of search strategies are described in Online Supplementary Appendix S2.

\section{Study Selection}

We included studies that reported associations between ethnicity and any of the prespecified outcomes indicative of severe COVID-19 disease in laboratory-confirmed SARS-CoV2 patients. Primary outcome is all-cause mortality. Secondary outcomes are hospitalisation, critical care admission, advanced respiratory support requirement (such as invasive mechanical ventilation (IMV), extracorporeal membrane oxygenation (ECMO)) and acute kidney injury (any severity or the need for acute renal replacement therapy). In particular, we excluded studies that reported on infection rates alone. Interventional trials, case reports, commentaries and articles from news media were excluded. In this article, we use the term 'ethnicity' to describe both 'ethnicity' and 'race' (Supplementary Methods S1).

\section{Data Extraction and Quality Assessment}

A custom spreadsheet was developed and piloted by AR and AT prior to use for data extraction; this recorded study characteristics (name, date, publication status), aim, location, setting, participant characteristics including age, sex, ethnicity (White, Black, Asian, Hispanic, mixed or missing data), body mass index, smoking status, comorbidities (hypertension, diabetes, chronic kidney disease, pulmonary disease, cardiovascular disease, asthma, COPD, coronary artery disease, heart failure, cancer) and whether the study collected any data on socioeconomic factors. Raw outcome data were extracted, along with any adjusted and unadjusted risk estimates (hazard ratios (HRs), relative risks (RRs) and odds ratios (ORs)).

The Newcastle-Ottawa Scale (NOS) and the modified NOS by Modesti were used to assess risk of bias for longitudinal cohort studies and ecological studies respectively $[9,10]$. A maximum score of 9 is possible, with 7 and above being regarded as low risk of bias. Pairs of investigators (AR, AT and LK) independently carried out data extraction and assessed risk of bias. A third investigator resolved any disagreement through discussion. Details of the data extraction form and NOS can be found in Online Supplementary Methods S3 and S4.

GRADE framework was used to assess the quality of evidence, and certainty in the adjusted risk estimates for each ethnicity-outcome association [11]. Observational studies provided high-quality evidence for prognostic factors, and are down-rated for risk of bias, inconsistency, imprecision, indirectness and publication bias or up-rated for strong evidence of association $(\mathrm{RR}>2$ or $<0.5)$ [12].

\section{Data Synthesis and Analysis}

Meta-analysis was carried out if two or more longitudinal cohort studies compared risk of outcomes in Black, Asian or Hispanic ethnic group with White participants (reference group) for each outcome. When multiple articles studied the same patient cohort, we would only use the cohort study reporting the largest number of events in the meta-analysis to avoid double-counting; this assumes a longer follow-up time. Ethnicity-outcome associations in COVID-19 patients were assessed using DerSimonian-Laird random-effects meta-analyses in the R package meta, as we expect heterogeneity across prognostic studies. If the risk estimates were not reported but raw data available, we calculated RRs and 95\% confidence intervals for cohort and single arms of casecontrolled studies. RRs were used as they do not overestimate risks, and outcomes were not rare in most studies [13]. ORs were converted to RRs using the Zhang and $\mathrm{Yu}$ formula, as developed in $\mathrm{R}$ (orsk) for the purpose of meta-analysis if outcome was $>10 \%$ [14]. OR was assumed to approximate RR if the outcome in the study was rare.

Meta-analyses of unadjusted and adjusted risk estimates were carried out separately for each ethnicity-outcome association, with pooled $\log (\mathrm{HR})$ and $\log (\mathrm{RR})$ calculated separately $[15,16]$. We present two sets of adjusted estimates: (1) adjusted for age and sex; (2) age, sex and at minimum one 
comorbidity. For ease of readership and interpretation, $\log (\mathrm{HR})$ and $\log (\mathrm{RR})$ were converted to HR and RR in the texts. Study heterogeneity was evaluated by $I^{2}$ statistic and visual inspection of the forest plots. Forest plots are displayed in Online Supplementary Results S3.

Reasons for heterogeneity were explored; Subgroups analyses stratified by locations (UK or USA) or risks of bias (NOS $<7$ or $\geq 7$ ) were conducted, including assessments of interaction. As a post hoc analysis, we calculated pooled risk estimates after excluding small studies $(n<100)$. Funnel plots were examined for publication bias and evaluated for asymmetry using Begg's rank correlation test as data from observational studies were likely to have asymmetric distribution [17]. All statistical analyses were performed in RStudio (version 1.3.959).

\section{Results}

The literature search retrieved 5706 articles on the databases, including 1043 duplicates. Additional 296 preprint articles were screened. Titles and abstract screening excluded 4883 articles, and full-text examination excluded 45 articles. Manual searching, reference tracking and citation tracking yielded a further 27 articles. Study selection process is illustrated according to PRISMA flowchart (Fig. 1).

There were 72 articles (59 cohort and 13 ecological studies) included for qualitative synthesis; 41 (57\%) studies were peerreviewed publications at the time of screening. Studies were conducted in the USA (54 studies), the UK (15), Brazil (one) and Israel (one). There was one multinational study based across the USA, Canada and Spain. Fifty-one studies assessed ethnic disparity in risks of mortality, 21 on hospitalisation, 18 on ICU admission, 18 on IMV and 8 on kidney failure. Adjusted analyses were carried out by 40 of 59 cohort studies and 5 of 13 ecological studies. The median NOS score was 7 (interquartile range 6-8, range 4-9). Twenty-four (33\%) studies had a NOS score of less than 7, mainly due to failure to control for age or comorbidities or non-representative study sample such as pregnant women, paediatric, cancer or transplant patients (Supplementary Results S1.1-2).

Of the 59 cohort studies, including one case-controlled study in which suitable data was extracted from one of its arms, there were $17,950,989$ participants: $11,502,289(64 \%)$ were White; 383,303 (2.1\%) were Black; 1,055,396 (5.9\%) were Asian; 15,439 (0.086\%) were Hispanic; 4,596,081 $(26 \%)$ had missing ethnicity data. Study characteristics are summarised in Table 1. Forty-five studies were included for meta-analysis. Summary of pooled risk estimates could be found in Table 2. A high level of heterogeneity was observed between studies. Publication bias was not detected (Supplementary Results S4).

\section{Mortality}

Fifty-one studies reported ethnicity-aggregated mortality data, including 38 cohort studies comprising 17,501,820 participants $(63 \%$ White, $2.1 \%$ Black, 6.0\% Asian, $0.069 \%$ Hispanic, $2.9 \%$ others and $26 \%$ missing ethnicity data). Total sample sizes were more than 100 participants $(n>100)$ in 26 of 28 (93\%) cohort studies included in the meta-analysis.

Pooled estimates from hazard ratios and relative risks generally showed similar magnitude, direction of effect and statistical significance. In the unadjusted analyses, compared to White ethnicity, all-cause mortality risk was similar in Black (RR: 0.96 [95\% CI: 0.83-1.08], $I^{2}=88, k=25$ ) and Asian (RR: 0.99 [95\% CI: $0.85-1.16$ ], $I^{2}=84, k=14$ ) but significantly reduced in Hispanic ethnicity (RR: 0.69 [95\% CI: 0.57$\left.0.84], I^{2}=76, k=11\right)$. Age- and sex-adjusted mortality risks were significantly elevated for Black (HR: 1.38 [95\% CI: 1.09-1.75], $I^{2}=94, k=5$ ) and Asian (HR: 1.42 [95\% CI: 1.15-1.75], $I^{2}=87, k=3$ ), but not for Hispanic (RR: 1.14 [95\% CI: $0.93-1.40$ ] $I^{2}=0, k=3$ ). Further adjustment for comorbidities attenuated these associations, rendering associations non-significant; HR (Black): 0.95 [95\% CI: 0.72-1.25], $I^{2}=79, k=4$; HR (Asian): 1.17 [95\% CI: $0.84-1.63$ ] $I^{2}=73$, $k=3$; HR (Hispanic): 0.94 [95\% CI: 0.63-1.44], $I^{2}=89, k=2$.

Subgroup analysis by location showed a consistent trend towards greater mortality risk estimates in UK ethnic minorities, but difference was not significant. Subgrouping by risk of bias did not demonstrate different effects (Supplementary Results S2).

Ten cohort studies, not included in meta-analysis, echoed similar findings, and did not support ethnicity as an independent risk factor for poor COVID-19 outcomes. Two studies reported non-significant difference in unadjusted mortality risk in Black (vs. non-Black) [18, 19]. Four studies (three unadjusted, one adjusted analyses) did not find an increase in mortality risk amongst non-White ethnicity [20-23]. Four studies reported lower mortality risk in Hispanic patients compared to that in non-Hispanic patients; two reported significant unadjusted analysis, and none reported significant ageadjusted analyses [24-27].

There were 12 US-based ecological design studies, utilising publicly available dataset to draw an indirect association between ethnicity and COVID-19 outcomes. Eight studies illustrated that counties with a greater proportion of African-Americans have higher rates of countylevel COVID-19 hospitalisations and deaths [28-32], even after adjusting for a combination of county-level characteristics such as age, poverty, comorbidities, healthcare access and geography [33-36]. One study did not find an increased mortality risk in Asian ethnicity after adjustment for age and geography [35]. Instead, a higher proportion of Asian population was a protective factor to counties [32]. Three studies reported increased age-adjusted mortality in 
Fig. 1 Study selection process

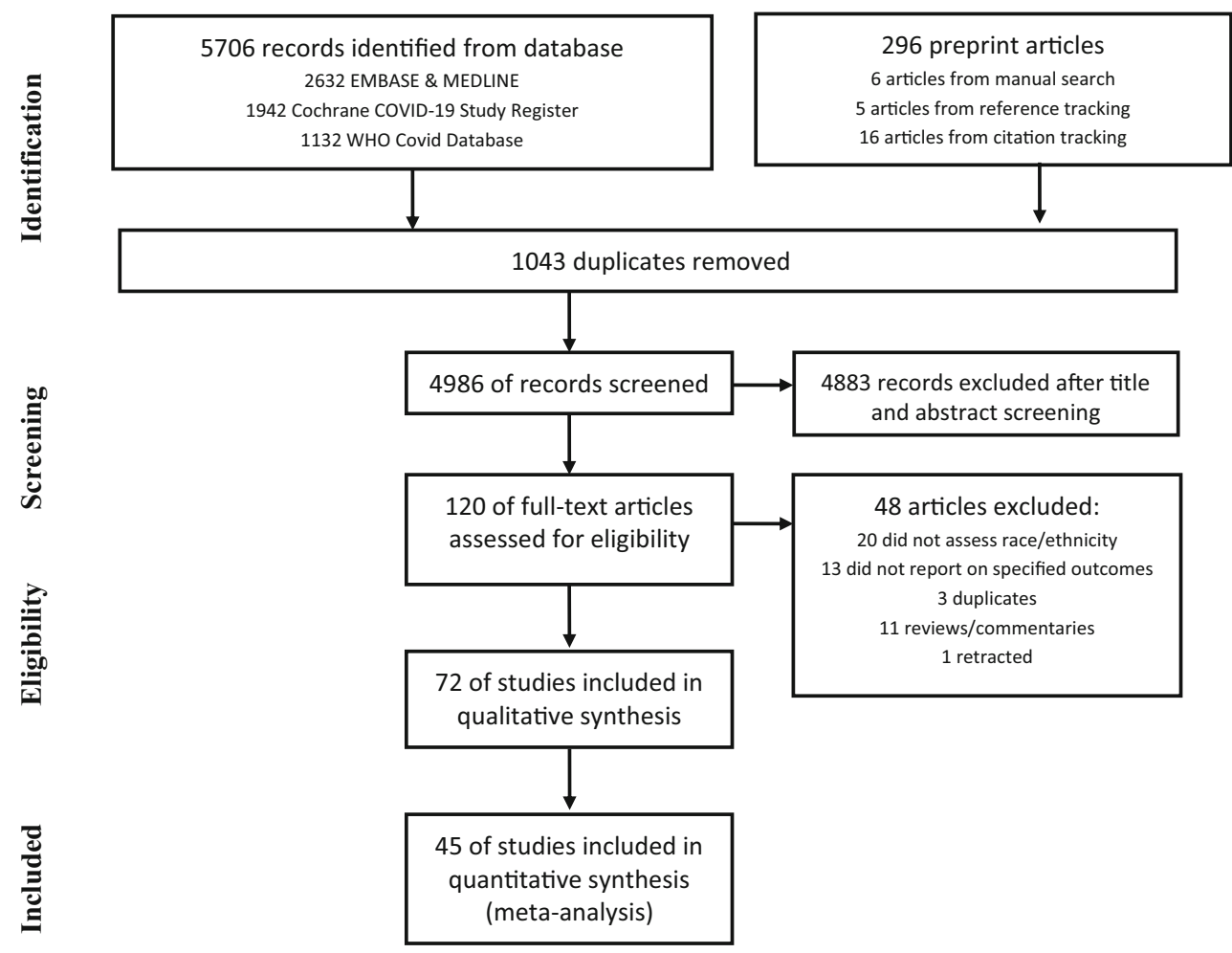

the Hispanic population [32, 35-37]. One study noted that ethnic segregation correlated with risks of COVID-19 death [38]. One UK study showed that Black African, Black Caribbean, Pakistani, Bangladeshi and Indian ethnic groups had higher age-standardised and geographyadjusted mortality ratio [39].

\section{Hospitalisation}

Twenty-one studies assessed hospitalisation risk in different ethnic groups. There were 20 cohort studies comprising 428,000 patients $(90 \%$ White, $4.5 \%$ Black, 3.4\% Asian, $1.6 \%$ Hispanic, $3.0 \%$ others and $0.19 \%$ missing ethnicity data); 14 articles were suitable for meta-analysis. Only one had a small sample size $(n<100)$.

Compared to White ethnicity, crude unadjusted risk of hospitalisation was significantly higher in Black (RR: 1.68 [95\% CI: $1.28-2.20], I^{2}=98, k=13$ ), but similar for Asian (RR: 1.13 [95\% CI: 0.78-1.66], $I^{2}=95, k=8$ ) and Hispanic (RR: 1.00 [95\% CI: 0.95-1.06], $I^{2}=0, k=8$ ) ethnicity. Ageand sex-adjusted risk was significantly raised in Black (RR: 2.23 [95\% CI: $1.54-3.19], I^{2}=92, k=5$ ) and Hispanic (RR: 1.49 [95\% CI: $1.28-1.75], I^{2}=0, k=2$ ), but not Asian (RR: 1.16 [95\% CI: 0.64-2.08], $I^{2}=82, k=3$ ) ethnicity. Weakening of association was noted after further adjustment for comorbidities: Black (RR: 1.40 [95\% CI: 0.93-2.12], $I^{2}=$ 95, $k=4$ ), Asian (RR: 1.04 [95\% CI: 0.99-1.11], $I^{2}=0, k=3$ ) and Hispanic (RR: 1.24 [95\% CI: 1.02-1.52], $I^{2}=75, k=3$ ).
Five studies considered further socioeconomic factors in their analysis and showed that adjusting for socioeconomic factors could reduce the disparity in hospitalisation risk [40-44].

Subgroup analysis showed strongly significant interaction $p$ value between UK and US subgroups. The hospitalisation risk of Black and Asian was markedly higher in the UK. For Black ethnicity, RR is 5.47 [95\% CI: 2.51-12.06] in UK studies vs. RR 1.36 [95\% CI: 1.08$1.72]$ in US studies with a $p$ value of 0.0008 . For Asian ethnicity, RR is 2.95 [95\% CI: 1.55-5.53] in UK studies vs. RR 0.90 [95\% CI: 0.82-1.66] in US studies with a $p$ value of 0.0003 . Subgrouping by location was not possible for Hispanic ethnicity. Subgrouping by NOS did not show significant interaction, although there was a trend towards greater risk in studies with lower NOS (Supplementary Results S2).

Seven studies were not suitable for meta-analyses. In a descriptive unadjusted analysis, three studies reported no differences between the number of Hispanic and non-Hispanic patients [25], Black and non-Black patients [18] and between White and BAME patients [20]. Despite no significant differences, ethnic minorities were still disproportionately represented in COVID-19 admissions [45, 46]. Two other studies reported increased adjusted risks of hospitalisation in nonWhite ethnic groups in both the USA and the UK [42, 47]. One New York study showed that rates of COVID-19 hospitalisations were higher in areas with greater proportion of ethnic minorities [28]. 
Table 1 Summary of study characteristics

\begin{tabular}{|c|c|c|c|c|c|c|}
\hline & Overall & Mortality & Hospitalisation & ICU admission & Kidney failure & IMV \\
\hline No. of studies & 72 & 51 & 21 & 18 & 8 & 18 \\
\hline Peer-reviewed & 41 & 27 & 13 & 11 & 4 & 14 \\
\hline \multicolumn{7}{|l|}{ Study location } \\
\hline UK & 15 & 11 & 5 & 5 & 1 & 1 \\
\hline USA & 55 & 39 & 16 & 13 & 7 & 17 \\
\hline Others & 4 & 3 & 0 & 0 & 0 & 0 \\
\hline Ecological studies & 13 & 13 & 1 & 0 & 0 & 0 \\
\hline Cohort studies & 59 & 38 & 20 & 18 & 8 & 18 \\
\hline $\begin{array}{c}\text { No of participants in } \\
\text { cohort studies }(N)\end{array}$ & $17,950,989^{*}$ & $17,501,820$ & 428,000 & 30,301 & 21,999 & 16,862 \\
\hline White, $n(\%)$ & $11,502,289(64)$ & $11,093,885(63)$ & $385,113(90)$ & $13,611(45)$ & $8334(38)$ & $6958(41)$ \\
\hline Black & $383,303(2.1)$ & $372,020(2.1)$ & $19,395(4.5)$ & $9830(32)$ & $8347(38)$ & $6914(41)$ \\
\hline Asian & $1,055,396(5.9)$ & $1,045,189(6.0)$ & $14,726(3.5)$ & $1289(4.3)$ & $1137(5.2)$ & $865(5.1)$ \\
\hline Hispanic & $15,439(0.086)$ & $12,148(0.069)$ & $6888(1.6)$ & $2405(7.9)$ & $2007(9.1)$ & $657(3.9)$ \\
\hline Mixed or others & $529,953(2.9)$ & $514,340(2.9)$ & $11,520(3.0)$ & $1873(6.2)$ & $2854(13)$ & $941(5.6)$ \\
\hline Missing data & $4,596,081(26)$ & $4,594,472(26)$ & $834(0.19)$ & $1438(4.7)$ & $610(2.8)$ & $718(4.3)$ \\
\hline \multicolumn{7}{|l|}{$\begin{array}{l}\text { Newcastle-Ottawa scale } \\
\text { points, median (IQR), range }\end{array}$} \\
\hline $\begin{array}{l}\text { Number of studies with } \\
\text { NOS } \geq 7 \text { points, } k(\%)\end{array}$ & $48 / 72(67)$ & $36 / 51(71)$ & $13 / 21(62)$ & $14 / 18(78)$ & $8 / 8(100)$ & $14 / 18(78)$ \\
\hline Overall & $7(6-8), 4-9$ & $7(6-8), 4-9$ & $8(6-8), 4-9$ & $8(7.25-9), 6-9$ & $8.5(7.75-9), 7-9$ & $8(7.25-9), 6-9$ \\
\hline Selection of study groups (max 4) & $4(3-4), 2-4$ & $4(3-4), 2-4$ & $4(3-4), 2-4$ & $4(3-4), 3-4$ & $4(3.75-4), 2-4$ & $4(3-4), 3-4$ \\
\hline Comparability of groups (max 2) & $1.5(0-2), 0-2$ & $1(0-2), 0-2$ & $2(0.25-2), 0-2$ & $2(1.25-2), 0-2$ & $2(2-2), 0-2$ & $2(1.25-2), 0-2$ \\
\hline Outcome (max 3$)$ & $3(2-3), 1-3$ & $3(2-3), 1-3$ & $2(2-3), 1-3$ & $3(2-3), 2-3$ & $3(3-3), 2-3$ & $3(2.25-3), 1-3$ \\
\hline
\end{tabular}

*One study was excluded as data derived from the same cohort [41, 42]. Studies may separate ethnicity from race; in such cases, Hispanic and other races are not mutually exclusive

\section{Critical Care Admission}

Eighteen studies assessed ethnicity as a risk factor for ICU admission, comprising 30,301 participants (45\% White, 32\% Black, 7.9\% Asian, $7.9 \%$ Hispanic and $4.7 \%$ with missing ethnicity data). Subgroup analyses by location or NOS scores were not possible as ten of 11 studies included in metaanalyses were US-based, and all have NOS $\geq 7$.

Compared to White ethnicity, the unadjusted risks of ICU admission were significantly raised in Black (RR: 1.51 [95\% CI: 1.11-2.04], $I^{2}=94, k=10$ ), similar in Asian (RR: 1.02 [95\% CI: 0.51-2.22], $I^{2}=71, k=4$ ) and lower in Hispanic ethnicity (RR: 0.89 [95\% CI: $0.75-1.05], I^{2}=0, k=6$ ). However, pooled estimates for Asian included three USbased studies and one UK study. The largest study was UKbased and reported an increased risk for Asian ethnicity [48]. The other three studies were US-based studies, relatively smaller sample sizes, showing non-significant associations.

Risk of ICU admission for Black ethnicity was attenuated to non-significant level after adjusting for age and sex (RR: 1.39 [95\% CI: $0.85-2.27$ ] $I^{2}=69, k=3$ ), and further after accounting for comorbidities (RR: 1.31 [95\% CI: 0.842.03], $\left.I^{2}=91, k=4\right)$. There was inadequate data for meta- analysis for Asian ethnicity; one study reported significantly increased age- and sex-adjusted risk of ICU Admission for Asian ethnicity [49]. Only two studies reported age-, sexand comorbidity-adjusted analysis for Hispanic ethnicity, showing a non-significant association with ICU admission (RR: 0.93 [95\% CI: $0.74-1.16], I^{2}=0, k=2$ ).

Seven studies were not suitable for meta-analysis. Five UK-based studies reported over-representation of the BAME communities in ICU cohorts [20, 21, 46, 50,51], with two reporting higher age-adjusted risk for BAME [20, 21]. On the other hand, two US studies did not find a significant difference in risk of ICU admission between Black and non-Black study participants $[18,19]$.

\section{Respiratory Failure}

Eighteen cohort studies comprising 16,862 participants (41\% White, $41 \%$ Black, 5.1\% Asian, 3.9\% Hispanic and 4.3\% missing ethnicity data) reported ethnicity-aggregated data on the need for advanced respiratory support, i.e. invasive mechanical ventilation (IMV). Thirteen studies were suitable for meta-analysis. 
Table 2 Summary estimates for mortality with $\log$ (hazard ratio) [95\% confidence interval], $I^{2}$ and number of studies ( $k$ ), or log (relative risk) [95\% confidence interval], $I^{2}$ and number of studies

\begin{tabular}{|c|c|c|c|}
\hline Ethnicity (White as reference) & Univariate & Multivariate $^{\dagger}$ & Multivariate \\
\hline \multicolumn{4}{|l|}{ Mortality } \\
\hline Black vs. White, $\log (\mathrm{HR})$ & $-0.17[-0.63 ; 0.30], 94, k=4$ & $0.32[0.09 ; 0.56]^{*}, 94, k=5$ & $-0.05[-0.33 ; 0.22], 79, k=4$ \\
\hline Black vs. White, $\log (\mathrm{RR})$ & $-0.05[-0.18 ; 0.08], 88, k=25$ & $0.25[0.03 ; 0.46]^{*}, 12, k=5$ & $0.06[-0.05 ; 0.17], 60, k=6$ \\
\hline Asian vs. White, $\log (\mathrm{HR})$ & $0.01[-0.31 ; 0.33], 82, k=4$ & $0.35[0.14 ; 0.56]^{*}, 87, k=3$ & $0.16[-0.18 ; 0.49], 73, k=3$ \\
\hline Asian vs. White, $\log (\mathrm{RR})$ & $-0.01[-0.16 ; 0.15], 84, k=14$ & $0.42[0.04 ; 0.80]^{*}, 0, k=2$ & NA \\
\hline Hispanic vs. White, log (HR) & NA & NA & $-0.06[-0.47 ; 0.35], 89, k=2$ \\
\hline Hispanic vs. White, $\log (\mathrm{RR})$ & $-0.36[-0.56 ;-0.17]^{*}, 76, k=11$ & $0.13[-0.08 ; 0.34], 0, k=3$ & NA \\
\hline \multicolumn{4}{|l|}{ Hospitalisation } \\
\hline Black vs. White, $\log (\mathrm{RR})$ & $0.52[0.25 ; 0.79]^{*}, 98, k=13$ & $0.80[0.43 ; 1.16]^{*}, 92, k=5$ & $0.34[-0.07 ; 0.75], 95, k=4$ \\
\hline Asian vs. White, $\log (\mathrm{RR})$ & $0.13[-0.25 ; 0.50], 95, k=8$ & $0.15[-0.44 ; 0.73], 82, k=3$ & $0.04[-0.01 ; 0.10], 0, k=3$ \\
\hline Hispanic vs. White, $\log (\mathrm{RR})$ & $0.00[-0.05 ; 0.05], 0, k=8$ & $0.40[0.25 ; 0.56]^{*}, 0, k=2$ & $0.22[0.02 ; 0.42]^{*}, 75, k=3$ \\
\hline \multicolumn{4}{|l|}{ Intensive care unit admission } \\
\hline Black vs. White, $\log (\mathrm{RR})$ & $0.41[0.11 ; 0.71]^{*}, 94, k=10$ & $0.33[-0.16 ; 0.82], 69, k=3$ & $0.27[-0.18 ; 0.71], 91, k=4$ \\
\hline Asian vs. White, $\log (\mathrm{RR})$ & $0.02[-0.67 ; 0.70], 71, k=4$ & NA & NA \\
\hline Hispanic vs. White, $\log (\mathrm{RR})$ & $-0.12[-0.28 ; 0.05], 0, k=6$ & NA & $-0.07[-0.29 ; 0.15], 0, k=2$ \\
\hline \multicolumn{4}{|l|}{ Invasive mechanical ventilation } \\
\hline Black vs. White, $\log (\mathrm{RR})$ & $0.25[-0.11 ; 0.60], 85, k=10$ & $0.34[0.12 ; 0.56]^{*}, 0, k=3$ & $0.21[-0.51 ; 0.92], 91, k=3$ \\
\hline Asian vs. White, $\log (\mathrm{RR})$ & $0.33[0.07 ; 0.59]^{*}, 14, k=4$ & $0.43[0.16 ; 0.70]^{*}, 0, k=2$ & NA \\
\hline Hispanic vs. White, $\log (\mathrm{RR})$ & $-0.12[-0.37 ; 0.14], 0, k=6$ & NA & $0.01[-0.17 ; 0.19], 0, k=2$ \\
\hline \multicolumn{4}{|l|}{ Acute kidney injury } \\
\hline Black vs. White, $\log (\mathrm{RR})$ & $0.30[0.04 ; 0.56]^{*}, 92, k=5$ & NA & $-0.47[-0.12 ; 1.07], 95, k=2$ \\
\hline Asian vs. White, $\log (\mathrm{RR})$ & $-0.17[-0.35 ; 0.01], 0, k=2$ & NA & NA \\
\hline Hispanic vs. White, $\log (\mathrm{RR})$ & NA & NA & NA \\
\hline
\end{tabular}

*Statistically significant; ${ }^{\dagger}$ Adjusted for age and sex; ${ }^{\ddagger}$ Adjusted for age, sex and comorbidities. $N A$ data not available. All forest plots are available in Online Supplementary Results S3

In unadjusted analyses, compared to White ethnicity, risk of IMV was higher in Black (RR: 1.28 [95\% CI: 0.90-1.81], $I^{2}=85 \%, k=10$ ) and Asian (RR: 1.39 [95\% CI: $\left.1.07-1.80\right]$, $I^{2}=14, k=4$ ) but lower in Hispanic ethnicity (RR: 0.89 [95\% CI: 0.69-1.15], $I^{2}=0, k=6$ ). Age- and sex-adjusted risks were significantly high for Black (RR: 1.40 [95\% CI: 1.13-1.75], $I^{2}=0, k=3$ ) and Asian ethnicity (RR: 1.54 [95\% CI: $1.17-$ $2.02], I^{2}=0, k=2$ ); There was only one study reporting nonsignificantly raised age- and sex-adjusted risk of IMV in Hispanic patients [52]. After full adjustment, the associations were attenuated and non-significant in Black (RR: 1.23 [95\% CI: $0.61-2.51], I^{2}=91, k=3$ ) and Hispanic ethnicity (RR: 1.01 [95\% CI: 0.84-1.21], $I^{2}=0, k=2$ ). One study reported a non-significantly lower age-, sex- and comorbidity-adjusted risk of IMV in Asian [53].

Subgrouping by location was not possible as all but one study were US-based. In subgroup analysis by risk of bias, unadjusted risks of IMV for Asian and Hispanic ethnicity were reported higher by studies with low risk of bias, albeit there was no significant interaction of $p$ value between subgroups. Inclusion of studies with high-risk bias did not affect the direction or the statistical significance of the overall pooled estimate, although it attenuated the magnitude.

Results from studies that were not suitable for metaanalysis further reiterated that ethnicity was not associated with risk of intubation. Four studies showed that Black ethnicity was not independently associated with IMV, or a composite of IMV or death [18, 19, 26, 54]. Two studies reported similar rates of IMV in Hispanic and other ethnicities [25, 26], although one study found non-significantly lower risk of IMV or death in Hispanics [54]. One found non-White ethnicity to be more likely to require high-flow oxygen support or IMV, albeit this was statistically non-significant [47]. We did not find any studies reporting association between ethnicity and ECMO.

\section{Kidney Failure}

Eight studies comprising 21,999 participants (38\% White, $38 \%$ Black, 5.2\% Asian, 9.1\% Hispanic, 2.8\% missing ethnicity data) investigated the association between ethnicity and acute kidney injury (AKI); all had low risk of bias and seven 
were US-based studies. Five studies were included in the meta-analysis.

In an unadjusted analysis, Black ethnicity was at a significantly higher risk of AKI (RR: 1.35 [95\% CI: 1.04-1.76], $I^{2}=92 \%, k=5$ ). Two separate studies showed that this association remained significant after adjustment of age, sex and comorbidities, but pooled RR was non-significant (RR: 1.60 [95\% CI: $0.89-2.90], I^{2}=95 \%, k=2$ ) most likely due to only two studies being suitable. Three studies reported higher rates of Black patients requiring acute renal replacement therapy, although none showed significant association in adjusted analysis $[19,48,55]$.

Two studies reported lower unadjusted risk of AKI in Asian ethnicity (RR: 0.85 [95\% CI: 0.71-1.01], $I^{2}=0, k=$ 2). One study reported non-significantly lower adjusted risk of AKI in Asian ethnicity [56]. Two studies did not find an increased unadjusted risk of AKI in Hispanic ethnicity [25, 57]. There were no studies reporting adjusted risk of AKI for Hispanic ethnicities.

\section{Quality Assessment}

The level of evidence was high for Black ethnicity, but low for both Asian and Hispanic ethnicities (Table 3). The certainty in the risk estimates for Asian and Hispanic was down-rated for risk of bias and indirectness due to relatively low number of studies providing age-, sex- and comorbidity-adjusted association, and potential differences between study participants and target population. Detailed assessment is described in Supplementary Results S5.

\section{Discussion}

This systematic review and meta-analysis could not confirm ethnicity as an independent risk factor for poor outcomes in COVID-19 patients. Analyses of step-wise adjustments for covariates underlined important factors confounding ethnicity as a risk factor.
Interpretation of ethnicity-stratified data requires considerations of traditional risk factors for poor outcomes in COVID19. Unadjusted risk ratios crudely quantify risk disparities between different ethnicities, but may misrepresent the true association. For instance, a low unadjusted mortality risk in the Hispanic group was due to a relatively younger Hispanic population included in the studies. This contrasts with the elevated age- and sex-adjusted risks across several outcome measures. Attenuation of these risk estimates with further adjustment for comorbidities indicates that disparities could be partially attributed to a greater burden of comorbidities in ethnic minority groups.

Heterogeneity in the meta-analysis can be attributed to differences in the magnitude rather than the direction of effect. Substantial $I^{2}$ is expected in meta-analyses of large observational studies producing narrow confidence intervals [12]. Clinical heterogeneity is expected in risk estimates for Asians since Asian ethnicity is not a homogenous group, consisting of individuals from widely diverse origins such as Indian, Pakistani, Bangladeshi, Chinese and others. Subgrouping by location has provided context-specific risk estimates, whilst sacrificing precision for general applicability in public health policy decision-making. We therefore argue that a high level of heterogeneity has not limited the usefulness of the meta-analyses. Nevertheless, for this reason, we down-rate certainty of risk estimates for Asian and Hispanic ethnicity.

To our knowledge, this is the first meta-analysis quantifying the association between ethnicity and COVID-19 outcomes. Our finding is in keeping with findings from pandemic influenza refuting ethnicity as a risk factor [58]. A more recent study continued to echo findings from this meta-analysis [59]. Whilst this review did not support ethnicity as an independent risk factor, the evidence is consistent on the disproportionate representation of ethnic minorities in COVID-19 mortality and morbidity.

This study has clinical and public health implications. Findings from this review should inform decisions regarding risk stratification at work, shielding advice and future allocation of vaccinations. Given the low-to-high-quality evidence indicating that ethnicity is not an independent risk factor,

Table 3 Quality of evidence assessment. Age, sex and comorbidities adjusted risk estimates for each ethnicity-outcome association, and application of GRADE principles towards rating confidence in risk estimates

\begin{tabular}{|c|c|c|c|c|c|c|c|c|c|c|c|}
\hline $\begin{array}{l}\text { All- } \\
\text { cause } \\
\text { mortality }\end{array}$ & Hospitalisation & $\mathrm{ICU}$ & Ventilation & $\begin{array}{l}\text { AKI or } \\
\text { acute RRT }\end{array}$ & $\begin{array}{l}\text { Risk of } \\
\text { bias }\end{array}$ & Inconsistency & Imprecision & Indirectness & $\begin{array}{l}\text { Publication } \\
\text { bias }\end{array}$ & $\begin{array}{l}\text { Strong } \\
\text { association }\end{array}$ & GRADE \\
\hline Ntrl & Ntrl & Ntrl & Ntrl & $\uparrow$ & - & - & - & - & - & - & High \\
\hline Ntrl & Ntrl & NA & NA & NA & Down & - & - & Down & - & - & Low \\
\hline $\mathrm{Ntrl}$ & $\uparrow *$ & Ntrl & Ntrl & NA & Down & - & - & Down & - & - & Low \\
\hline
\end{tabular}

GRADE Grading of Recommendations Assessment, Development and Evaluation, Ntrl neutral association, NA not applicable. $\uparrow *$ Significant risk factor; $\uparrow$ Potential risk factor; non-significant risk ratio $>1.5$; risk ratio between 0.67 and 1.5 ; $\downarrow$ Potentially protective; non-significant risk ratio $<0.67$; $\downarrow *$ Significant protective 
COVID-19 risk assessment should only consider ethnicity in conjunction with other risk factors such as age or comorbidities. Nevertheless, public campaigns need to continue to target ethnic minority groups to reduce rates of infection and medical comorbidities amongst ethnic minorities; both appeared to be the driving forces behind the overrepresentation of racial minorities in COVID-19 mortality.

Our study has several strengths. Search strategy was comprehensive, covering a large number of published peer-reviewed and preprint articles. A further six of 31 preprint articles went on to be published in peer-review journals by $15 / 08 / 2020$. A large number of studies were included in the meta-analysis with only several small study sizes $(n<100)$; Omission of these studies in post hoc analysis did not affect the direction and statistical significance of analyses. Multiple indicators of poor outcomes, i.e. mortality, hospitalisation, ICU admission, intubation and kidney failure, were considered. Taken together, the balance of evidence weighs against ethnicity as an independent risk factor for poor outcomes in patients with laboratoryconfirmed SARS-CoV-2 infection. Lastly, separate pooling of unadjusted and adjusted risk estimates improves our understanding of ethnicity-outcome association.

Our study is limited by certain gaps in currently available evidence: substantial number of ethnicity data missing in studies; limited number of articles on socioeconomic factors as a confounding factor to ethnicity; minimal articles studying adjusted risks in Hispanic, Asian and native populations. There was a minimal involvement of paediatric patients in our meta-analysis, and so findings should not be extrapolated to paediatric patients. Given reports of association between SARS-CoV-2 and Kawasaki-like disease, this area needs to be explore further. We did not investigate whether ethnicity is associated with risk of SARS-CoV-2 infection as this relates to incidence rather than severity. Besides, this has been explored in a previous systematic review [6].

Despite these limitations, our rigorous study updates current evidence on the association between ethnicity and poor COVID-19 outcomes, and identifies gaps in evidence that future studies can work towards.

Supplementary Information The online version contains supplementary material available at https://doi.org/10.1007/s40615-020-00921-5.

Authors' Contributions AR, AT and LK are equally contributing co-first authors. All authors are equally involved in the conception, data collection, processing, analysis and manuscript writing. Statistical analysis is mainly carried out by AT.

\section{Compliance with Ethical Standards}

Conflict of interest The authors declare that they have no conflict of interest.
Reproducible Research Statement Study protocol: see "Method" and Supplement. Statistical code: see "Method." Data set: All included studies are publicly available; additional data are available upon request from corresponding author.

\section{References}

1. Wu Z, McGoogan JM. Characteristics of and important lessons from the coronavirus disease 2019 (COVID-19) outbreak in China. JAMA. American Medical Association. 2020;323:1239-42.

2. Yang J, Zheng Y, Gou X, Pu K, Chen Z, Guo Q, et al. Prevalence of comorbidities and its effects in coronavirus disease 2019 patients: a systematic review and meta-analysis. Int J Infect Dis. Elsevier B.V. 2020;94:91-5.

3. Bibbins-Domingo K. This time must be different: disparities during the COVID-19 pandemic. Ann Intern Med. 2020:M20-2247. https://doi.org/10.7326/M20-2247.

4. COVID-19: Data Summary - NYC Health. [Internet] Available from: https://www1.nyc.gov/site/doh/covid/covid-19-data.page. Accessed 8 Jul 2020.

5. Intensive Care National Audit \& Research Centre. ICNARC report on COVID-19 in critical care.

6. Pan D, Sze S, Minhas JS, Bangash MN, Pareek N, Divall P, et al. The impact of ethnicity on clinical outcomes in COVID-19: a systematic review. EClinicalMedicine Lancet Publishing Group. 2020;23:100404.

7. Khunti K, Singh AK, Pareek M, Hanif W. Is ethnicity linked to incidence or outcomes of covid-19? BMI. 2020;369:m1548. https:// doi.org/10.1136/bmj.m1548.

8. Moher D, Liberati A, Tetzlaff J, Altman DG, Altman D, Antes G, et al. Preferred reporting items for systematic reviews and metaanalyses: the PRISMA statement. PLoS Med. 2009;6:e1000097.

9. Wells G, Shea B, O'Connell D, Peterson J, Welch V, Losos M, et al. Ottawa Hospital Research Institute. Available from: http:// www.ohri.ca/programs/clinical epidemiology/oxford.asp. Accessed 22 Jun 2020.

10. Modesti PA, Reboldi G, Cappuccio FP, Agyemang C, Remuzzi G, Rapi S, et al. Panethnic differences in blood pressure in europe: a systematic review and meta-analysis. PLoS One. 2016;11(1): e0147601. https://doi.org/10.1371/journal.pone.0147601.

11. Iorio A, Spencer FA, Falavigna M, Alba C, Lang E, Burnand B, et al. Use of GRADE for assessment of evidence about prognosis: rating confidence in estimates of event rates in broad categories of patients. BMJ. 2015;350:h870. https://doi.org/10.1136/bmj.h870.

12. Foroutan F, Guyatt G, Zuk V, Vandvik PO, Alba AC, Mustafa R, et al. GRADE guidelines 28: use of GRADE for the assessment of evidence about prognostic factors: rating certainty in identification of groups of patients with different absolute risks. J Clin Epidemiol. Elsevier Inc.; 2020;121:62-70. Available from: https://oi.org/10. 1016/j.jclinepi.2019.12.023.

13. Ranganathan P, Aggarwal R, Pramesh CS. Common pitfalls in statistical analysis: odds versus risk. Perspect Clin Res. 2015;6(4): $222-4$.

14. Wang Z. Converting odds ratio to relative risk in cohort studies with partial data information. J Stat Softw. 2013;55. https://doi.org/10. 18637/jss.v055.i05.

15. Higgins J, Li T, Deeks J. Chapter 6: choosing effect measures and computing estimates of effect. In: Cochrane Handbook for Systematic Reviews of Interventions version 6.0. Cochrane Training. Chichester: John Wiley \& Sons; 2019.

16. Riley RD, Moons KGM, Snell KIE, Ensor J, Hooft L, Altman DG, et al. A guide to systematic review and meta-analysis of prognostic factor studies. BMJ. 2019;364:k4597. https://doi.org/10.1136/bmj. k4597. 
17. Begg CB, Mazumdar M. Operating characteristics of a rank correlation test for publication bias. Biometrics. 1994;50(4):1088-101.

18. Suleyman G, Fadel RA, Malette KM, Hammond C, Abdulla H, Entz A, et al. Clinical characteristics and morbidity associated with coronavirus disease 2019 in a series of patients in metropolitan Detroit. JAMA Netw Open. 2020;3:e2012270.

19. Gold JAW, Wong KK, Szablewski CM, Patel PR, Rossow J, Da Silva J, et al. Characteristics and clinical outcomes of adult patients hospitalized with COVID-19 - Georgia, March 2020. Morb Mortal Wkly Rep. 2020;69:545-50.

20. Kakkar DN, Dunphy DJ, Raza DM. Ethnicity profiles of COVID19 admissions and outcomes. J Infect. 2020;81(2):e110-e111. https://doi.org/10.1016/j.jinf.2020.05.059.

21. Galloway JB, Norton S, Barker RD, Brookes A, Carey I, Clarke $\mathrm{BD}$, et al. A clinical risk score to identify patients with COVID-19 at high risk of critical care admission or death: an observational cohort study. J Infect. 2020;81(2):282-288. https://doi.org/10. 1016/j.jinf.2020.05.064.

22. Field RE, Afzal I, Dixon J, Patel VR, Sarkar P, Marsh JE. Cohort profile: preliminary experience of 500 COVID-19 postive cases at a South West London District General Hospital. medRxiv. Cold Spring Harbor Laboratory Press; 2020.

23. Imam Z, Odish F, Gill I, O'Connor D, Armstrong J, Vanood A, et al. Older age and comorbidity are independent mortality predictors in a large cohort of 1305 COVID-19 patients in Michigan, United States. J Intern Med. 2020;288(4):469-76. https://doi.org/ 10.1111/joim.13119.

24. Lala A, Johnson KW, Januzzi JL, Russak AJ, Paranjpe I, Richter F, et al. Prevalence and impact of myocardial injury in patients hospitalized with COVID-19 infection. J Am Coll Cardiol. 2020;76:53346.

25. Antwi-Amoabeng D, Beutler BD, Awad M, Kanji Z, Mahboob S, Ghuman J, et al. Sociodemographic predictors of outcomes in COVID-19: examining the impact of ethnic disparities in Northern Nevada. medRxiv. 2020;

26. Palaiodimos L, Kokkinidis DG, Li W, Karamanis D, Ognibene J, Arora S, et al. Severe obesity, increasing age and male sex are independently associated with worse in-hospital outcomes, and higher in-hospital mortality, in a cohort of patients with COVID19 in the Bronx, New York. Metab Clin Exp. 2020;108:154262.

27. Okoh AK, Sossou C, Dangayach NS, Meledathu S, Phillips O, Raczek C, et al. Coronavirus disease 19 in minority populations of Newark, New Jersey. Int J Equity Health. 2020;19:93.

28. Wadhera RK, Wadhera P, Gaba P, Figueroa JF, Joynt Maddox KE, Yeh RW, et al. Variation in COVID-19 hospitalizations and deaths across New York City Boroughs. JAMA. 2020;323(21):2192-5. https://doi.org/10.1001/jama.2020.7197.

29. Khose S, Moore JX, Wang HE. Epidemiology of the 2020 pandemic of COVID-19 in the state of Texas: the first month of community spread. J Community Health. 2020;45:696-701.

30. Mahajan UM, Larkins-Pettigrew M. Racial demographics and COVID-19 confirmed cases and deaths: a correlational analysis of 2886 US counties. J Public Health (Oxf). 2020;fdaa070. https://doi. org/10.1093/pubmed/fdaa070.

31. Cyrus E, Clarke R, Hadley D, Bursac Z, Trepka MJ, Devieux JG, et al. The impact of COVID-19 on African American communities in the United States. medRxiv. 2020;

32. Abedi V, Olulana O, Avula V, Chaudhary D, Khan A, Shahjouei S, et al. Racial, economic and health inequality and COVID-19 infection in the United States. medRxiv. 2020.

33. Millett GA, Jones AT, Benkeser D, Baral S, Mercer L, Beyrer C, et al. Assessing differential impacts of COVID-19 on Black communities. Ann Epidemiol Elsevier BV. 2020;47:37-44.

34. Li AY, Hannah TC, Durbin J, Dreher N, McAuley FM, Marayati NF, et al. Multivariate analysis of factors affecting COVID-19 case and death rate in U.S. Counties: the significant effects of Black race and temperature. medRxiv. 2020;

35. Goldstein JR, Atherwood S. Improved measurement of racial/ ethnic disparities in COVID-19 mortality in the United States. MedRxiv. 2020. https://doi.org/10.1101/2020.05.21.20109116.

36. Gross CP, Essien UR, Pasha S, Gross JR, Wang S, Nunez-Smith M. Racial and ethnic disparities in population level COVID-19 mortality. medRxiv. 2020.

37. El Chaar M, King K, Galvez Lima A. Are black and Hispanic persons disproportionately affected by COVID-19 because of higher obesity rates? Surg Obes Relat Dis. 2020;16(8):1096-9. https://doi.org/10.1016/j.soard.2020.04.038.

38. Tian T, Zhang J, Hu L, Jiang Y, Duan C, Li Z, et al. Risk factors associated with mortality of COVID-19 in 2692 counties of the United States. medRxiv. 2020;

39. Aldridge RW, Lewer D, Katikireddi SV, Mathur R, Pathak N, Burns R, et al. Black, Asian and minority ethnic groups in England are at increased risk of death from COVID-19: indirect standardisation of NHS mortality data. Wellcome Open Res. 2020;5:88.

40. Price-Haywood EG, Burton J, Fort D, Seoane L. Hospitalization and mortality among Black patients and White patients with COVID-19. N Engl J Med. 2020;382:2534-43.

41. Lassale C, Gaye B, Hamer M, Gale CR, Batty GD. Ethnic disparities in hospitalisation for COVID-19 in England: the role of socioeconomic factors, mental health, and inflammatory and proinflammatory factors in a community-based cohort study. Brain Behav Immun. 2020;88:44-9. https://doi.org/10.1016/j.bbi.2020. 05.074.

42. Patel AP, Paranjpe MD, Kathiresan NP, Rivas MA, Khera A V. Race, Socioeconomic Deprivation, and Hospitalization for COVID-19 in English participants of a National Biobank. MedRxiv. 2020. https://doi.org/10.1101/2020.04.27.20082107.

43. Azar KMJ, Shen Z, Romanelli RJ, Lockhart SH, Smits K, Robinson $\mathrm{S}$, et al. Disparities in outcomes among COVID-19 patients in a large health care system in California. Health Aff. 2020;39:125362.

44. Gu T, Mack JA, Salvatore M, Sankar SP, Valley TS, Singh K, et al. COVID-19 outcomes, risk factors and associations by race: a comprehensive analysis using electronic health records data in Michigan Medicine. Medrxiv. 2020; Available from: https://doi. org/10.1101/2020.06.16.20133140.

45. Garg S, Kim L, Whitaker M, O'Halloran A, Cummings C, Holstein $\mathrm{R}$, et al. Hospitalization rates and characteristics of patients hospitalized with laboratory-confirmed coronavirus disease 2019 COVID-net, 14 states, March 1-30, 2020. Morb Mortal Wkly Rep. Department of Health and Human Services: 2020; 458-64. Available from: https://www.cdc.gov/mmwr/volumes/69/wr/ mm6915e3.htm. Accessed 15 Jul 2020.

46. COVID-19: review of disparities in risks and outcomes - GOV.UK [Internet]. PHE. [cited 2020 Jul 9]. Available from: https://www. gov.uk/government/publications/covid-19-review-of-disparitiesin-risks-and-outcomes.

47. Robilotti EV, Babady NE, Mead PA, Rolling T, Perez-Johnston R, Bernardes M, et al. Determinants of COVID-19 disease severity in patients with cancer. Nat Med 2020;26:1218-23. https://doi.org/10. 1038/s41591-020-0979-0.

48. Apea VJ, Wan YI, Dhairyawan R, Puthucheary ZA, Pearse RM, Orkin CM, et al. Ethnicity and outcomes in patients hospitalised with COVID-19 infection in East London: an observational cohort study. medRxiv. 2020.

49. Hajifathalian K, Kumar S, Newberry C, Shah S, Fortune B, Krisko $\mathrm{T}$, et al. Obesity is associated with worse outcomes in COVID-19; analysis of early data from New York City. Obesity. 2020;28:160612. 
50. Thomas B, Emily P, Amrit D, Tamas S. Insights into the epidemiology of the first wave of COVID-19 ICU admissions in South Wales-the interplay between ethnicity and deprivation. Preprints. Preprints; 2020. Available from: www.preprints.org. Accessed 15 Jul 2020.

51. Harman K, Verma A, Cook J, Radia T, Zuckerman M, Deep A, et al. Ethnicity and COVID-19 in children with comorbidities. Lancet Child Adolesc Health. Elsevier B.V. 2020;4:e24-5. Available from: www.thelancet.com/child-adolescent. Accessed $15 \mathrm{Jul} 2020$.

52. Kalligeros M, Shehadeh F, Mylona EK, Benitez G, Beckwith CG, Chan PA, et al. Association of obesity with disease severity among patients with coronavirus disease 2019. Obesity. 2020;28:1200-4.

53. Hur K, Price CPE, Gray EL, Gulati RK, Maksimoski M, Racette $\mathrm{SD}$, et al. Factors associated with intubation and prolonged intubation in hospitalized patients with COVID-19. Otolaryngol Head Neck Surg. SAGE Publications Inc. 2020;163:170-8. https:/doi. org/10.1177/0194599820929640.

54. Geleris J, Sun Y, Platt J, Zucker J, Baldwin M, Hripcsak G, et al. Observational study of hydroxychloroquine in hospitalized patients with COVID-19. NEJM. Massachussetts Medical Society; 2020 [cited 2020 Jul 15];382:2411-8. Available from: https://doi.org/ 10.1056/NEJMoa2012410.
55. Khan A, Chatterjee A, Singh S. Comorbidities and disparities in outcomes of COVID-19 among African American and White patients. medRxiv. 2020.

56. Hirsch JS, Ng JH, Ross DW, Sharma P, Shah HH, Barnett RL, et al. Acute kidney injury in patients hospitalized with COVID-19. Kidney Int. 2020;98:209-18.

57. Chan L, Chaudhary K, Saha A, Chauhan K, Vaid A, Baweja M, et al. Acute kidney injury in hospitalized patients with COVID-19. medRxiv. 2020.

58. Mertz D, Kim TH, Johnstone J, Lam PP, Science M, Kuster SP, et al. Populations at risk for severe or complicated influenza illness: systematic review and meta-analysis. BMJ. 2013;347:f5061. https://doi.org/10.1136/bmj.f5061.

59. Yehia BR, Winegar A, Fogel R, Fakih M, Ottenbacher A, Jesser C, et al. Association of race with mortality among patients hospitalized with coronavirus disease 2019 ( COVID-19) at 92 US hospitals. 2020;2019.

Publisher's Note Springer Nature remains neutral with regard to jurisdictional claims in published maps and institutional affiliations. 